\title{
La cartographie multi-scalaire d'un habitat sur un site accidenté : la Silla del Papa (Espagne)
}

\author{
Multi-scale mapping of a habitat on a rugged site: Silla del Papa (Spain)
}

\author{
Antoine Laurent ${ }^{1}$, Pierre Moret ${ }^{1}$, Jean-Marc Fabre ${ }^{1}$, Carine Calastrenc ${ }^{1}$, Nicolas Poirier ${ }^{1}$ \\ ${ }^{1}$ CNRS, UMR 5608 TRACES
}

\begin{abstract}
RÉSUMÉ. Dans le cadre du programme ANR Archeostraits, une cartographie de l'habitat du site archéologique de la Silla del Papa (sud de l'Espagne) a été entreprise. Elle est rendue complexe par la topographie combinant de forts dénivelés et une végétation persistante. De plus, les bâtiments de la fin de l'âge du fer sont ancrés à plusieurs niveaux dans les parois de barres rocheuses, ce qui rend inopérante une analyse architecturale traditionnelle à partir de planimétries en 2D. L'analyse 3D à plusieurs échelles, de l'environnement au sondage, demande une adaptation des outils d'acquisition 3D à cet espace. Trois technologies présentes au sein du laboratoire Traces générant des nuages de points denses ont été utilisées.

ABSTRACT. As part of the ANR Archeostraits program, a mapping of the archaeological remains of the site of Silla del Papa (Southern Spain) was undertaken. It is made complex by a rugged topography, with strong unevenness and a ground covered by persistent vegetation. In addition, Late Iron Age buildings are embedded in rock walls at several levels, making traditional architectural analysis ineffective with 2D planimetry. Multi-scale 3D analysis, from the surrounding territory to the excavation of a house, requires an adaptation of 3D acquisition tools. For this purpose, we have used three technologies developed in the Traces laboratory to generate dense point clouds.

MOTS-CLÉS. acquisition 3D, nuage de points dense, Lidar, topographie.

KEYWORDS. 3D acquisition, dense cloud, Lidar, topography.
\end{abstract}

Lien vers le poster:

https://sharedocs.huma-num.fr/wl/?id=CfY8DeTJHXaDmGmseGAZkmbR9u0RtUJt

Le site archéologique de la Silla del Papa se trouve à $4 \mathrm{~km}$ de la côte, sur le point le plus élevé d'une petite chaîne côtière, la Sierra de la Plata $(457 \mathrm{~m})$, qui ferme à l'ouest la baie de Bolonia. Le lieu présente trois atouts qui ne pouvaient qu'attirer les populations qui fréquentaient le Détroit : de formidables défenses naturelles formées par des affleurements rocheux presque verticaux ; les eaux abondantes d'une source pérenne située au pied de ces rochers ; enfin, une position dominante offrant des vues lointaines dans toutes les directions : au sud jusqu'à Tanger, au sud-est jusqu'au Djebel Moussa, à l'ouest jusqu'au cap Trafalgar.

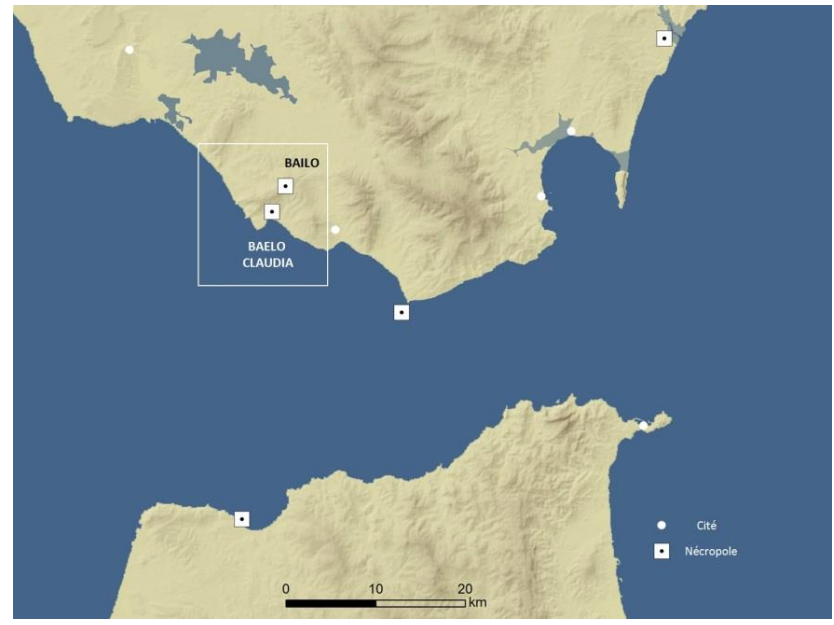

Figure 1. Localisation du site près du détroit de Gibraltar 
L'organisation spatiale du site est conditionnée par l'existence de deux barres rocheuses parallèles orientées nord-sud qui délimitent un étroit couloir de $420 \mathrm{~m}$ de long. La largeur de l'espace habitable entre les deux lignes de crête oscille entre 20 et $75 \mathrm{~m}$. Ces caractéristiques physiques rendent complexe l'acquisition des données spatiales. Pour acquérir et analyser le site archéologique de son environnement aux excavations, une approche multiscalaire a été envisagée à partir de trois technologies 3D.

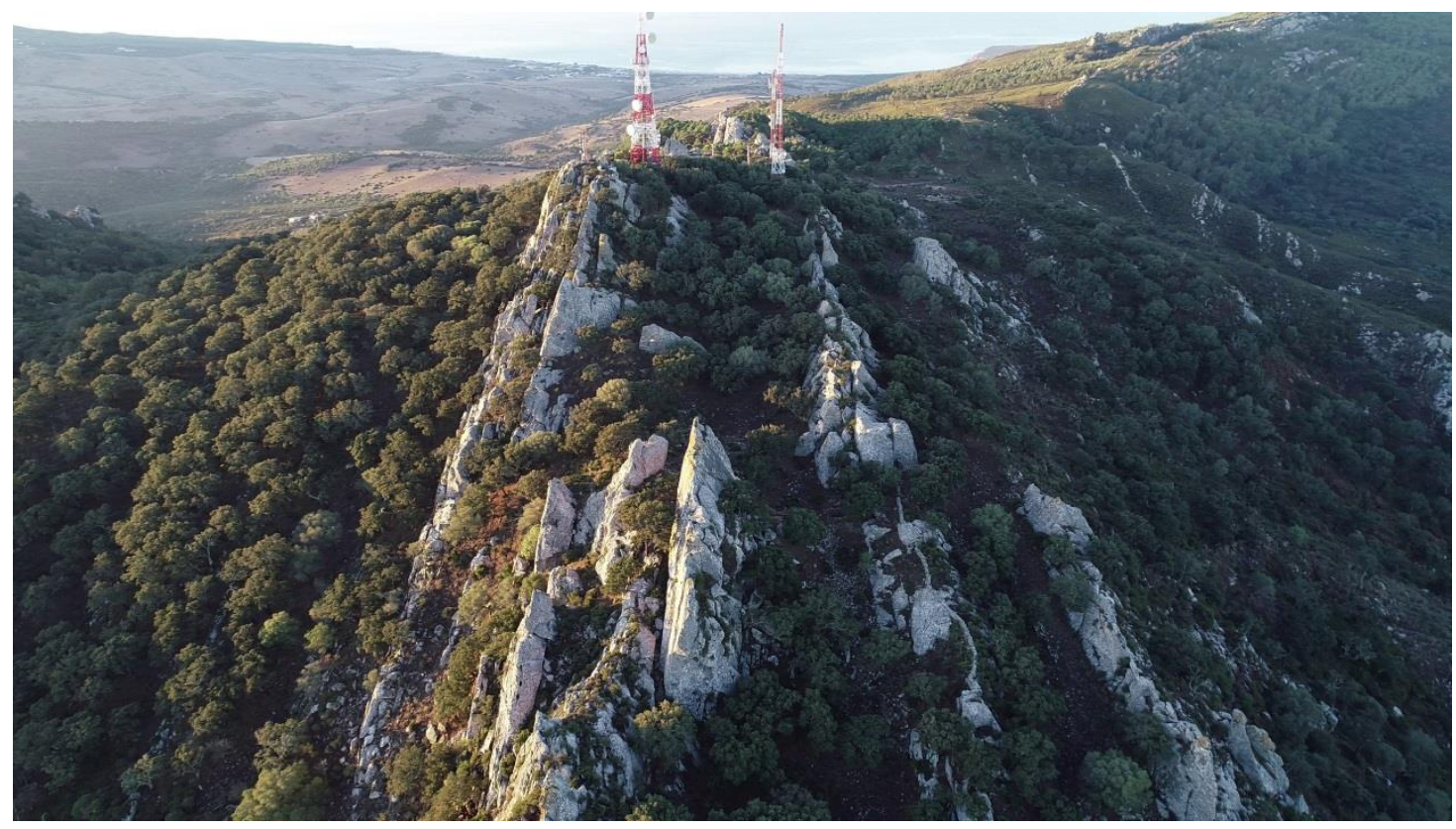

Figure 2. Le relief offre une protection naturelle

Les technologies d'acquisition 3D déployées sont le Lidar embarqué sur un drone (ALS), le scanner 3D (TLS) et la photogrammétrie (drone / perche / sol). Toutes trois fournissent, dans des formats interopérables, des nuages de points denses qui constituent le dénominateur commun permettant de combiner, croiser et compléter les modes d'acquisition à différentes échelles, chacune de ces technologies possédant une précision et une résolution de travail propres (du décimétrique au millimétrique).

\section{LIDAR aéroporté (ALS) : une cartographie 2D renouvelée}

Le Lidar aéroporté (modèle Yellowscan Mapper) a été employé à l'échelle du site (40 ha) pour obtenir une cartographie générale. Neuf vols ont été nécessaires pour couvrir l'habitat et les nécropoles à une altitude de 40 mètres. Après le nettoyage numérique de la végétation, 14 millions de points caractérisent le "sol". Ce squelette offre une précision à $15 \mathrm{~cm}$ permettant de réaliser un MNT avec une résolution à $25 \mathrm{~cm}$. La chaîne de traitement a privilégié les outils open-source afin de pouvoir répliquer le traitement sur d'autres terrains (LAStools, MCC- Lidar, Cloud Compare, CSF, SagaGis). La plus grande difficulté résidait dans le choix des seuils de calcul pour extraire le "sol". Au final, les espaces rocheux, les zones plates et les excavations ont été traités différemment.

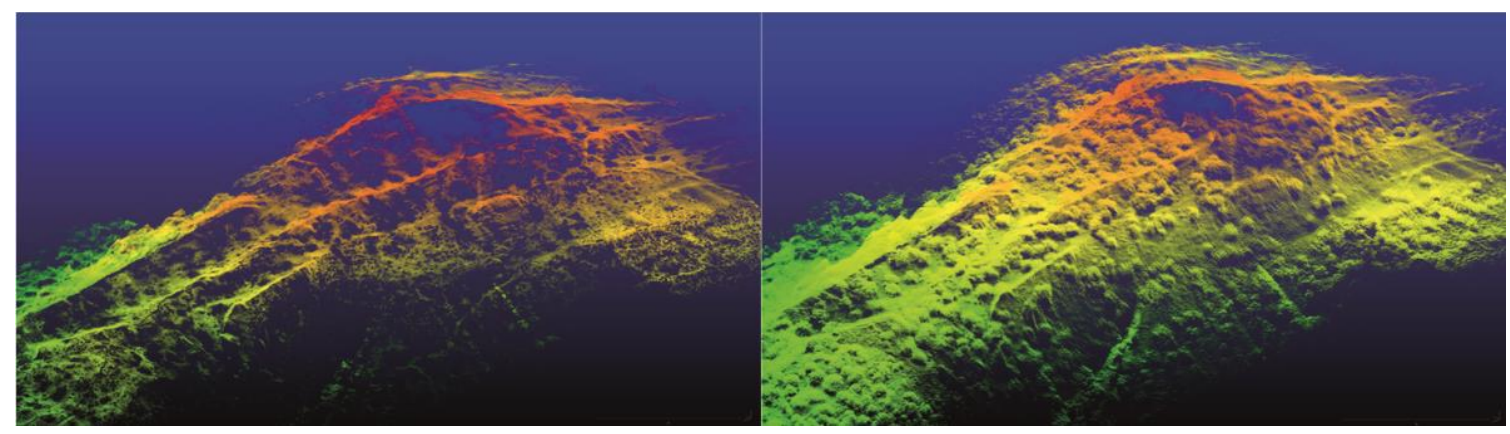

Figure 3. Du nuage Lidar brut jusqu'à l'extraction du « sol » 
Le résultat obtenu est un modèle numérique de terrain archéologique (MNT) qui occulte la végétation et les constructions contemporaines, tout en conservant les vestiges archéologiques. La cartographie du site devient ainsi plus précise et bénéficie d'une résolution bien supérieure à celle disponible à partir des données publiques de l'Institut Géographique National espagnol.

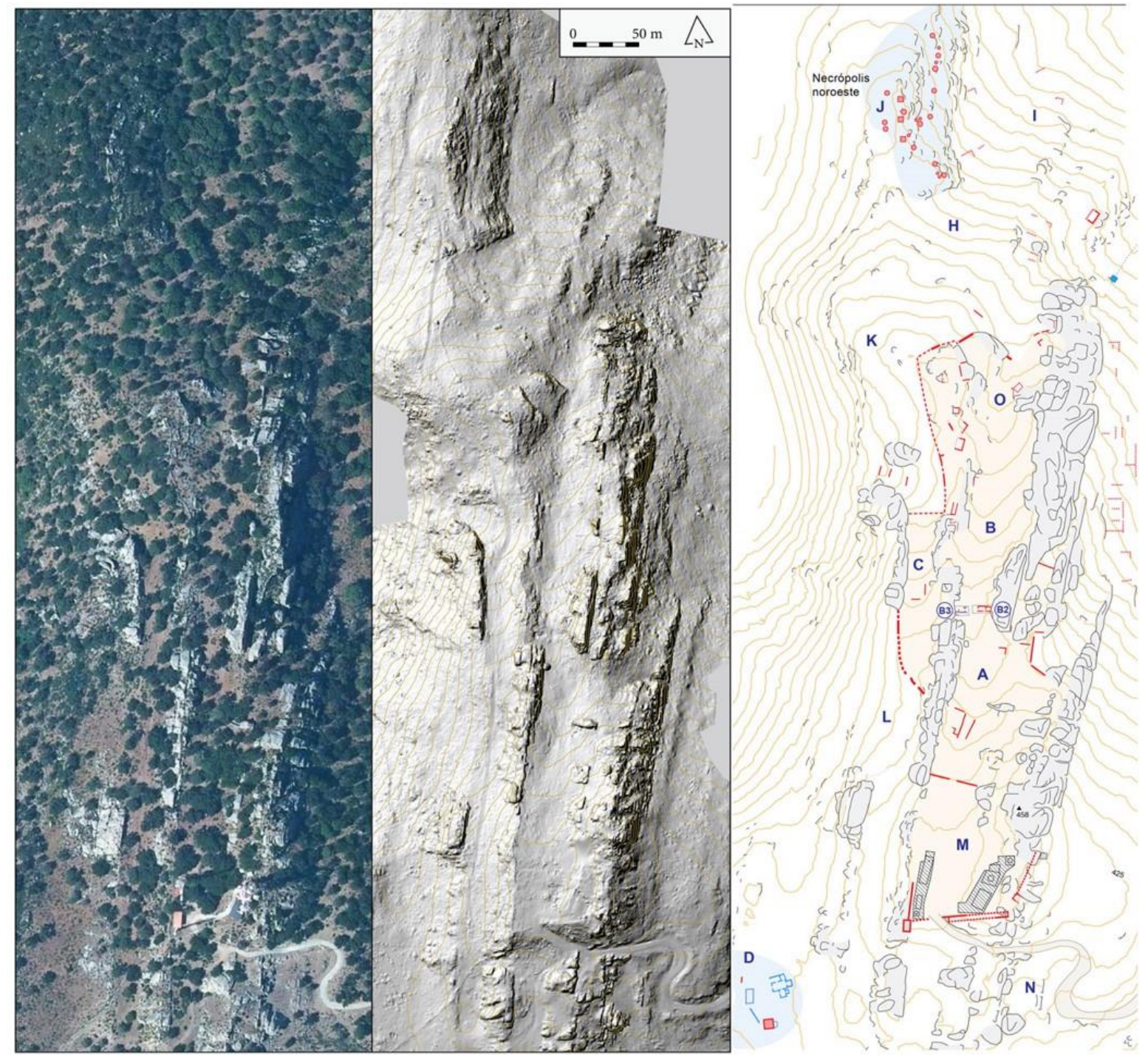

Figure 4. Du MNT Lidar au plan vectorisé en DAO

\section{Compléter et affiner le squelette 3D}

Le niveau de détail rendu nécessaire par certaines problématiques de recherche varie du décimétrique au millimétrique. Ainsi, pour l'étude des parois retaillées, la précision du scanner 3D a été privilégiée pour sa capacité à numériser dans un référentiel métrique local qui ne nécessite pas des outils de topographie classique. En scannant son environnement à $360^{\circ}$, il numérise les parties verticales insuffisamment renseignées par la fauchée du lidar aéroporté. L'assemblage des nuages de points a été réalisé avec l'outil «Iterative Closest Point» (ICP) dans Cloud Compare à partir des informations similaires. L'ICP compare les signatures 3D pour mettre les deux nuages de points dans le même référentiel. La résolution du MNT issu du scanner 3D est centimétrique et il vient compléter le squelette décimétrique généré par le Lidar. 


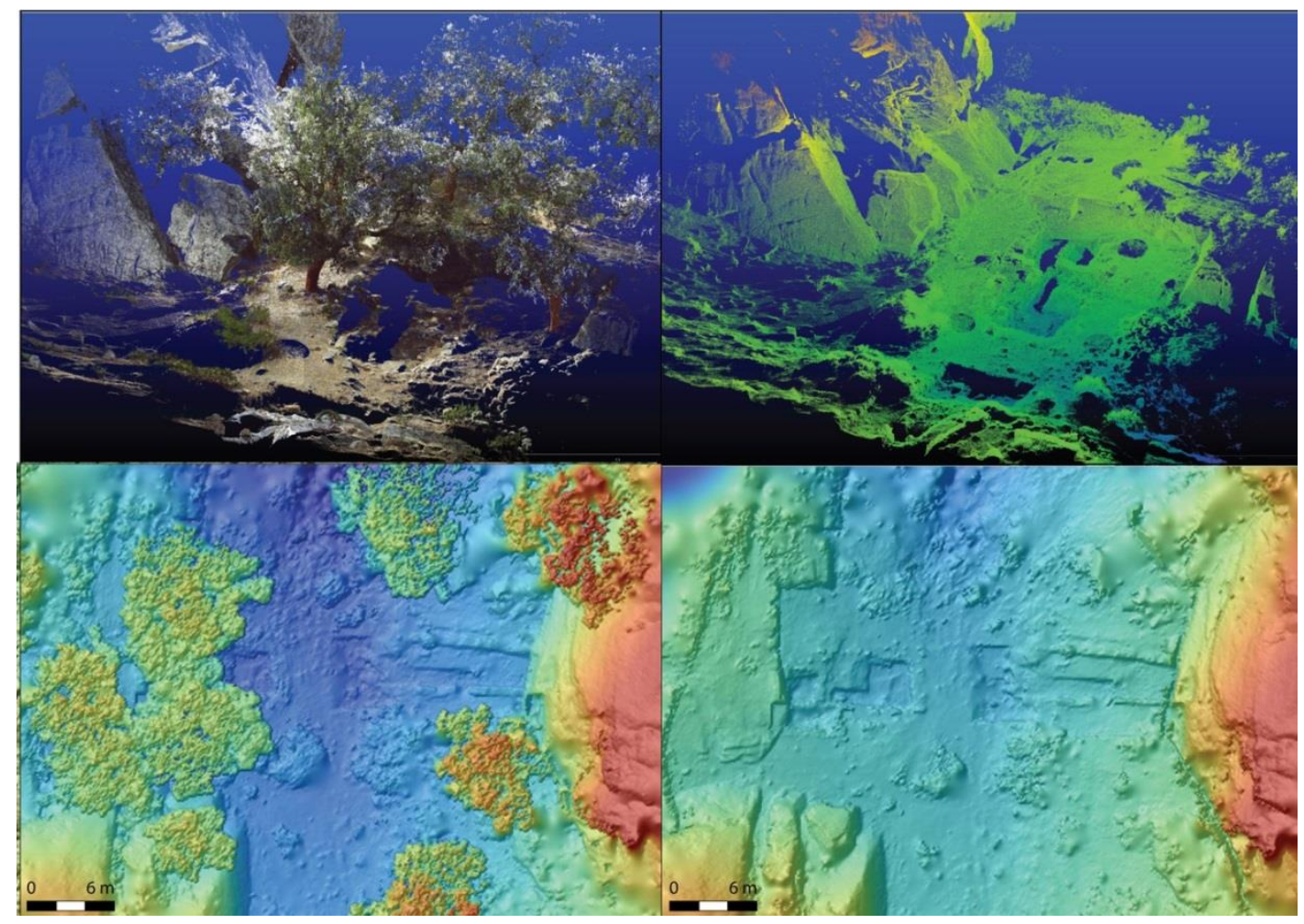

Figure 5. Le scanner 3D au cœur du site

\section{Une actualisation des données à chaque fouille}

Pour les espaces ouverts et les excavations, la photogrammétrie a été retenue pour sa rapidité d'acquisition et parce qu'elle s'appuie sur les références spatiales des fouilles. Son déploiement demande un faible investissement autorisant la multiplication des acquisitions aux différentes étapes des recherches (les fouilles de la zone D depuis 2014 : https://skfb.ly/6rNwZ). Dans les zones hors référentiel topographique, les nuages denses des photogrammétries ont pu être géolocalisées grâce aux données Lidar.

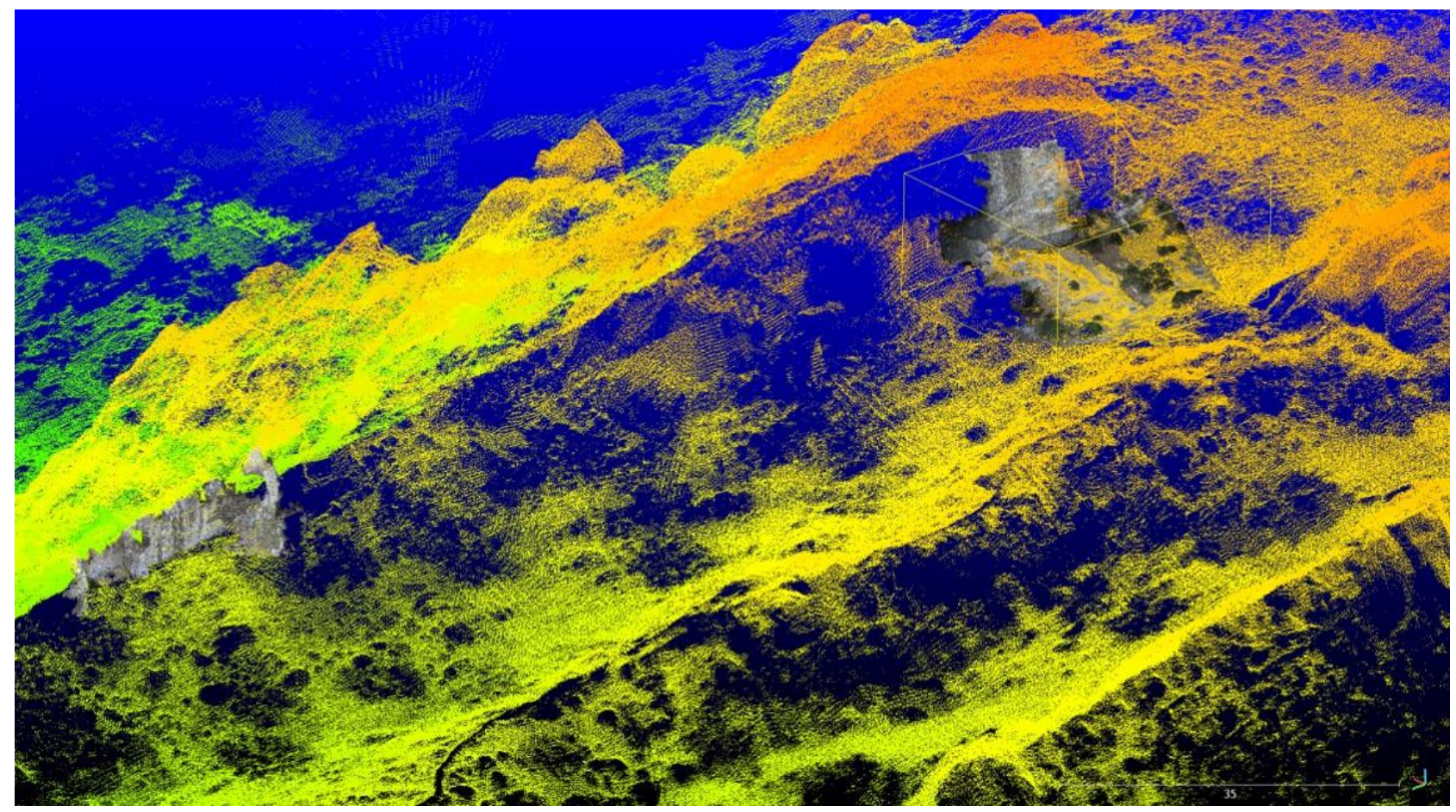

Figure 6. Assemblage d'une photogrammétrie sur le Lidar 
La manipulation de ce type de données 3D permet de prendre en compte une nouvelle dimension dans l'étude des sites qui enrichit le potentiel d'analyse spatiale des archéologues. Outre les difficultés topographiques d'un site et le choix de la technologie à appliquer, la génération de nuages de points denses rend possible le croisement des sources et la généralisation des chaînes de traitement.

\section{De l'analyse 3D au stockage pérenne}

L'ensemble des nuages de points produits a servi de support pour la réalisation des plans 2D du site archéologique, pour la production des coupes stratigraphiques, pour le SIG 3D (visualisation du Lidar en SIG : https://skfb.ly/YYUQ) ainsi que pour la réalisation de maillages destinés à la restitution à différentes échelles (exemple d'une proposition de restitution de la zone D au haut moyen-âge: https://skfb.ly/68EUP).

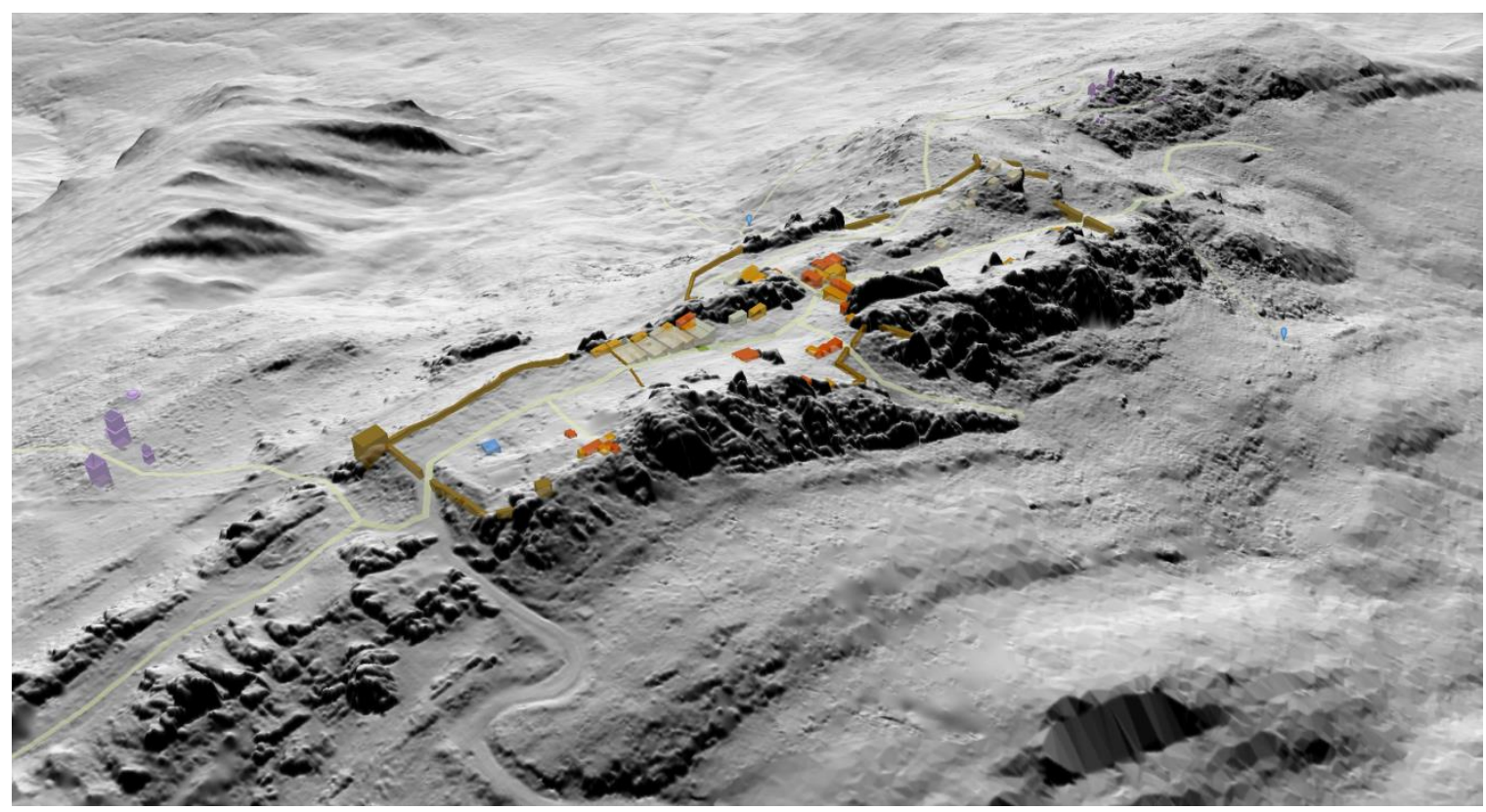

Figure 7. De l'acquisition 3D au SIG 3D et aux propositions de restitution

Cette étude multi-scalaire basée sur une combinaison de technologies pose au final les questions du stockage et de l'archivage des milliards de points produits. Toutes ces questions sont au centre du Consortium 3D SHS et de la très grande infrastructure de recherche Huma-Num. 


\section{Bibliographie et Webographie (dernière consultation le 20/10/18) :}

Moret, P., Fabre, J.-M., García, I., Prados, F., Constans, A. (2010), « La Silla del Papa (Tarifa, Cadix) : bilan de trois années de recherches », Pallas, 82, p. 441-463.

Moret, P., Prados, F. (2014), «Les deux Baelo : du site perché protohistorique au site portuaire romain sur la rive nord du détroit de Gibraltar », dans L. Mercuri, R. González y F. Bertoncello (éd.), Implantations humaines en milieu littoral méditerranéen : facteurs d'installation et processus d'appropriation de l'espace (Préhistoire, Antiquité, Moyen Âge), Antibes, p. 137-148.

Moret, P., Prados, F., Fabre, J.-M., García, I. et al. (2017), «La Silla del Papa: hábitat y necrópolis (campañas 20142016) », Mélanges de la Casa de Velázquez, 47 (1), p. 51-73.

https://blogs.univ-tlse2.fr/archeostraits/ - Blog du projet Archeostraits

https://www.yellowscan-lidar.com/ - Site du matériel Lidar embarqué

https://www.danielgm.net/cc/ - Site de Cloud Compare

https://shs3d.hypotheses.org/ - Le carnet de recherche du Consortium 3D SHS

https://www.huma-num.fr/ - La TGIR des humanités numériques

https://sketchfab.com/archeomatique - Plateforme de partage de modèle 3D 